Service social

\title{
Action collective et démocratie locale. Les mouvements urbains montréalais, par Pierre Hamel, Montréal, les Presses de l’Université de Montréal, 1991, 239 pages.
}

\section{Jean Panet-Raymond}

Volume 41, numéro 3, 1992

Intervenir en contexte d'autorité

URI : https://id.erudit.org/iderudit/706592ar

DOI : https://doi.org/10.7202/706592ar

Aller au sommaire du numéro

Éditeur(s)

École de service social de l'Université Laval

ISSN

1708-1734 (numérique)

Découvrir la revue

Citer ce compte rendu

Panet-Raymond, J. (1992). Compte rendu de [Action collective et démocratie locale. Les mouvements urbains montréalais, par Pierre Hamel, Montréal, les Presses de l'Université de Montréal, 1991, 239 pages.] Service social, 41(3), 133-134. https://doi.org/10.7202/706592ar d'utilisation que vous pouvez consulter en ligne.

https://apropos.erudit.org/fr/usagers/politique-dutilisation/ 
M. Matte porte, quant à elle, sur la question du REER et du régime de pension du conjoint.

Supporté par une jurisprudence importante, parsemé de notes explicatives et de tableaux, enrichi de plusieurs annexes, ce $37^{\mathrm{e}}$ recueil de textes du Service de la formation permanente (SFP) du Barreau du Québec renferme une variété de points de vue. Si le recueil se démarque par sa diversité, cette variété s'estompe toutefois devant l'objectif commun à toutes les conférences qu'il rassemble : informer davantage sur le droit familial contemporain. Psychologues, sociologues, travailleurs sociaux ou simples citoyens intéressés par le droit familial, tous trouveront enseignements et information en parcourant cet ouvrage.

En somme, c'est un guide très bien construit qui permet $d^{\prime}$ acquérir rapidement une compréhension multidimensionnelle du droit familial dans le contexte de 1992.

René AUCLAIR

École de service social Université Laval

Note : Les auteurs des textes sont Jean-Marie Fortin, Dominique Goubau, Marie-Christine Laberge, André M. Matte, Jean-Marc Neault, Élisabeth Pinard, Danielle Richer et Jean-Pierre Senécal.

\section{ACTION COLLECTIVE ET DÉMOCRATIE LOCALE. LES MOUVEMENTS URBAINS MONTRÉALAIS}

Pierre Hamel,

Montréal, Les Presses de l'Université de Montréal, 1991, 239 pages.

Dès le premier chapitre, l'auteur annonce le pari majeur de sa démarche, soit de mener de front la réflexion théorique et l'étude concrète des mouvements sociaux sur la scène urbaine montréalaise. Lourd pari en effet qui comportait des risques, car la lecture de ce livre très dense et très fouillé sur le plan théorique en est d'autant plus difficile ; le fil conducteur de la démarche théorique croise celui des illustrations des mouvements sociaux. Cela amène des failles dans la démonstration théorique et dans la description des pratiques. Le livre s'adresse donc à un public qui connaît les grands courants de pensée sur les mouvements sociaux et les pratiques des mouvements sociaux depuis 1960.

Cet avertissement fait, ce livre est un effort intéressant et stimulant pour comprendre le sens et la portée de l'engagement des acteurs dans les 
mouvements sociaux ainsi que l'impact de ces mouvements sur la scène locale et, plus particulièrement, sur le renouvellement de la démocratie locale à Montréal. L'auteur précise son objet d'étude comme étant « ...l'ensemble des actions menées par des associations bénévoles autour des enjeux urbains sur la scène locale » (p. 27). C'est pourquoi il emploie l'expression de mouvement urbain. Il y inclut 1) les groupes qui agissent sur le plan de la production et de l'organisation de l'espace urbain, 2) les groupes dont l'action porte sur les équipements et services collectifs et 3) les groupes « qui agissent relativement au développement local » en vue d'améliorer les conditions de vie par la création d'emplois, la formation et la stimulation de I'entrepreneurship (les corporations de développement économique communautaire, CDEC) (p. 25). Bien que l'objet soit donc limité à ces groupes montréalais et souvent à partir de données de recherches datant du début des années 80, les analyses peuvent être utiles et adaptées à l'ensemble des mouvements sociaux contemporains.

Sur le thème particulier des rapports entre les mouvements urbains et I'État, I'auteur fait une bonne analyse de l'évolution des rapports depuis I'affrontement systématique des années 70 jusqu'à I' « approche pragmatique » des années 80 et 90 . Sans apporter d'éléments vraiment originaux, il souligne avec justesse la contribution des mouvements à l'élaboration de politiques sociales, à la reconnaissance de la place du communautaire, mais aussi à l'intégration sociale par l'acceptation de jouer le jeu de la concertation. Cela ne va pas sans perdre une marge d'autonomie que la fragilité du financement ne peut compenser. Les CDEC illustrent bien cette approche pragmatique en acceptant les partenariats avec les réseaux public et privé.

La contribution sans doute la plus originale et intéressante dans cette analyse est le chapitre 7, qui propose une lecture individualiste de l'action collective. Les intérêts individuels des acteurs ont sans doute été trop longtemps considérés comme un sujet tabou. L'auteur apporte un regard rafraîchissant en montrant le lien entre les motivations et les apprentissages individuels et l'engagement dans I'action collective. La démocratie locale prend un sens nouveau à la lumière de cette analyse que les vieux militants auraient intérêt à lire attentivement.

Enfin, l'auteur conclut que les mouvements urbains ont eu un impact important en revalorisant la vie de quartier et en stimulant I'apprentissage individuel et collectif de la démocratie locale montréalaise. L'arrivée du RCM au pouvoir en 1986 en est un signe tangible, bien que partiel, malgré les déceptions de plusieurs par rapport aux attentes de décentralisation par la création des Comités de quartier. En cela, considère l'auteur, sans vouloir tomber dans une apologie, les mouvements urbains sont crédibles et légitimés, et ils ont atteint des objectifs majeurs.

Jean PANET-RAYMOND 